\title{
Characteristics of Cognitive Behavioral Therapy for Older Adults Living in Residential Care: Protocol for a Systematic Review
}

Phoebe Chan ${ }^{1}$; Sunil Bhar ${ }^{1}$, BA (Hons), MA (Clin Psych), PhD; Tanya E Davison ${ }^{2}$, BSc (Hons), DPsych (Clin); Colleen Doyle ${ }^{3}$, BA (Hons), PhD; Bob G Knight ${ }^{4}$, PhD; Deborah Koder ${ }^{5}$, BSc (Psych) (Hons), MClinPsych, PhD; Kenneth Laidlaw ${ }^{6}$, MA (Hons), MPhil (Clin Psych), PhD; Nancy A Pachana ${ }^{7}$, BA (Psych), MA (Psych), PhD (Clin Psych); Yvonne Wells ${ }^{8}, \mathrm{PhD}$; Viviana M Wuthrich ${ }^{9}$, BPsych (Hons), MPsych (Clin), PhD

${ }^{1}$ Department of Psychological Sciences, Swinburne University of Technology, Melbourne, Australia

${ }^{2}$ School of Health Sciences, Swinburne University of Technology, Melbourne, Australia

${ }^{3}$ Faculty of Health Sciences, Australian Catholic University, Melbourne, Australia

${ }^{4}$ School of Psychology and Counselling, University of Southern Queensland, Toowoomba, Australia

${ }^{5}$ Royal Prince Alfred Hospital, Sydney, Australia

${ }^{6}$ Department of Clinical Psychology, Norwich Medical School, University of East Anglia, Norwich, United Kingdom

${ }^{7}$ School of Psychology, The University of Queensland, Brisbane, Australia

${ }^{8}$ Lincoln Centre for Research on Ageing (Australian Institute for Primary Care and Ageing), La Trobe University, Bundoora, Australia

${ }^{9}$ Centre for Emotional Health, Department of Psychology, Macquarie University, Sydney, Australia

\section{Corresponding Author:}

Phoebe Chan

Department of Psychological Sciences

Swinburne University of Technology

PO Box 218

Hawthorn

Melbourne, Vic 3122

Australia

Phone: 61392148371

Fax: 61398196857

Email: phoebechan@swin.edu.au

\section{Abstract}

Background: The prevalence rates of depressive and anxiety disorders are high in residential aged care settings. Older adults in such settings might be prone to these disorders because of losses associated with transitioning to residential care, uncertainty about the future, as well as a decline in personal autonomy, health, and cognition. Cognitive behavioral therapy (CBT) is efficacious in treating late-life depression and anxiety. However, there remains a dearth of studies examining CBT in residential settings compared with community settings. Typically, older adults living in residential settings have higher care needs than those living in the community. To date, no systematic reviews have been conducted on the content and the delivery characteristics of CBT for older adults living in residential aged care settings.

Objective: The objective of this paper is to describe the systematic review protocol on the characteristics of CBT for depression and/or anxiety for older adults living in residential aged care settings.

Methods: This protocol was developed in compliance with the recommendations of the Preferred Reporting Items for Systematic Review and Meta-Analysis Protocols (PRISMA-P). Studies that fulfill the inclusion criteria will be identified by systematically searching relevant electronic databases, reference lists, and citation indexes. In addition, the PRISMA flowchart will be used to record the selection process. A pilot-tested data collection form will be used to extract and record data from the included studies. Two reviewers will be involved in screening the titles and abstracts of retrieved records, screening the full text of potentially relevant reports, and extracting data. Then, the delivery and content characteristics of different CBT programs of the included studies, where available, will be summarized in a table. Furthermore, the Downs and Black checklist will be used to assess the methodological quality of the included studies. 
Results: Systematic searches will commence in May 2018, and data extraction is expected to commence in July 2018. Data analyses and writing will happen in October 2018.

Conclusions: In this section, the limitations of the systematic review will be outlined. Clinical implications for treating late-life depression and/or anxiety, and implications for residential care facilities will be discussed.

Trial Registration: PROSPERO 42017080113; https://www.crd.york.ac.uk/PROSPERO/display_record.php?RecordID=80113 (Archived by WebCite at http://www.webcitation.org/70dV4Qf54)

Registered Report Identifier: RR1-10.2196/9902

(JMIR Res Protoc 2018;7(7):e164) doi: 10.2196/resprot.9902

\section{KEYWORDS}

cognitive behavioural therapy; older adults; residential care; delivery; characteristics; systematic review

\section{Introduction}

Older adults living in residential care settings have a high prevalence rate of depression and anxiety disorders. A systematic review of studies involving aged care residents from North America, Europe, Middle East, Australia, New Zealand, Africa, and Asia [1] reported that the prevalence of a major depressive disorder ranged from $4.8 \%$ (13/270) to $23.5 \%$ $(12 / 51)$, whereas the prevalence of depressive symptoms ranged from $14 \%(99 / 708)$ to $81.8 \%$ (113/138). Another systematic review that examined the prevalence of anxiety in older adults living in residential aged care [2] found that the prevalence of anxiety disorders ranged from $3.2 \%$ (31/966) to $20 \%$ (20/100), whereas the prevalence of clinically significant anxiety symptoms ranged from $6.5 \%$ (3/46)to $58.4 \%$ (118/202). The most common anxiety disorders among aged care residents were generalized anxiety disorder and specific phobias.

The high prevalence of depression and anxiety in residential care settings may be attributed to several factors, including losses (eg, social connections and personal possessions) and changes (eg, lifestyle and health) involved in transitioning to living in a residential care facility $[1,3,4]$. Other factors associated with depression and anxiety in older adults living in residential care include multiple chronic health problems, chronic pain, functional impairment in basic activities of daily living (eg, bathing), functional impairment in instrumental activities (eg, managing finances), sensory impairments (eg, vision and hearing), cognitive decline, loneliness, negative life events, lack of social support, perceived inadequacy of care, perceived inability to master and control external environment, a low sense of purpose in life, and low perceived autonomy [5-7]. Another contributing factor could be the patterns of interactions between nursing staff and residents of care facilities. Baltes et al [8,9] reported that residents developed learned dependency when the social environment provided consistent and immediate support for dependent self-care behaviors (eg, residents who did not attempt to eat by themselves would likely receive immediate help from staff members, whereas those who attempted to eat independently would not receive praise, encouragement, or attention). Thus, learned dependency could have a negative impact on residents' self-image and sense of control; subsequently, this could affect their psychological well-being.
To date, several approaches (eg, psychotherapy, pharmacological interventions, and music therapy) have been used to treat late-life depression and anxiety [10]. The efficacy of psychotherapeutic interventions, particularly cognitive behavioral therapies (CBT), has been demonstrated in several systematic reviews and meta-analyses conducted in the community and residential settings. CBT represents an approach that focuses on identifying and improving maladaptive behavioral and thinking patterns to assist clients in achieving goals. CBT includes a wide range of cognitive and behavioral techniques and is structured and goal-oriented.

Focusing on community-dwelling older adults, Wilson et al [11] reported that the efficacy of CBT in treating late-life depression was equivalent to or better than that of active control interventions (eg, visual imagery and education). Similarly, summarizing findings across community-based samples, Hendriks et al [12] reported that CBT was markedly more effective than waiting list and active control conditions (eg, usual care and supportive psychotherapy) in reducing anxiety symptoms in older adults diagnosed with anxiety disorders. In addition, a more recent meta-analysis [13] reported that CBT was markedly more effective in reducing anxiety symptoms in community-dwelling older adults compared with treatment as usual or being on a waiting list.

In a meta-analysis of the outcomes of psychotherapy for aged care residents, Cody and Drysdale [14] reported that psychotherapies were effective in reducing the symptoms of depression. In addition, they found that the effect of psychotherapies was comparable to that reported in pharmacotherapy trials with depressed older adults. Despite a lack of systematic reviews specifically examining CBT in residential care settings, studies such as those conducted by Anderson et al [15] and by Blair and Bird [16] found that CBT was effective and feasible for reducing depressive symptoms in older adults living in residential care.

Given the presence of multiple medical comorbidities and functional and cognitive decline, older adults in residential care might have different needs, clinical presentations, and perceptions of and responses to psychotherapies compared with community-dwelling older adults. In addition, the complexity of presentations might entail unique implementation models requiring interdisciplinary teamwork, sustainability of the intervention, flexibility of the environment, and support of the organization [17]. Specific programs, such as the Group, 
Individual, and Staff Therapy (GIST) [18] and the Behavioral Activities Intervention (BE-ACTIV) [19], have been developed for residential care settings. Nevertheless, no systematic review has been conducted on techniques and delivery characteristics of CBT when employed in residential care facilities. Such a systematic review is essential because it provides crucial information for the development of future CBT-based programs for residents in aged care. As highlighted by Kishita and Laidlaw [20] and Blair and Bird [16], identifying components of CBT programs that are specific to this population (eg, logistical issues such as how to approach residents, group size, timing, and duration of sessions) could help enhance treatment accessibility, acceptability, and outcomes. Moreover, by identifying the content of such protocols, that is, the strategies and techniques used to assist residents, clinicians may be better prepared to address the concerns of older adults living in residential aged care settings.

\section{Methods}

\section{Objectives}

This systematic review aims to describe the delivery and content characteristics of CBT for depression and anxiety for older adults living in residential aged care settings. This review adopts a broad definition of "older adults," those aged $\geq 55$ years.

\section{Protocol and Registration}

This protocol was developed to comply with the recommendations of the Preferred Reporting Items for Systematic Review and Meta-Analysis Protocols (PRISMA-P) [21]. This systematic review will follow the guidelines of the Preferred Reporting Items for Systematic Reviews and Meta-Analyses (PRISMA) [22]. The review has been registered with the International Prospective Register of Systematic Reviews (PROSPERO, CRD 42017080113).

\section{Eligibility Criteria for the Review}

\section{Participants}

In this review, those studies will be included, in which (1) participants were, at least, 55 years old, (2) were living in residential care (see definition below), and (3) standardized, valid measures (self-report questionnaire, observer rating, or clinical interview) were used to record the diagnosis and severity of depressive or anxiety disorders and symptom ratings of depression or anxiety. Samples of participants might include those living with dementia or mild cognitive impairment. Furthermore, studies that recruited participants aged $<55$ years but reported separate results for participants aged $\geq 55$ years will be included.

\section{Intervention}

The target intervention is CBT for depressive symptoms, depressive disorders, anxiety symptoms, or anxiety disorders. Consistent with other studies [12,14], the types of psychotherapies considered to be CBT include behavioral therapy (including behavioral activation and exposure-based interventions), cognitive therapy, CBT, problem-solving therapy, rational emotive behavioral therapy, and mindfulness- and acceptance-based cognitive and behavioral therapies. Of note, studies that focused only on non-CBT interventions (eg, psychodynamic therapies, interpersonal therapies, and systemic therapies) or on psychological problems other than depression and anxiety will be excluded from this review. Moreover, studies will be excluded if their primary aim was to reduce disruptive behaviors associated with dementia or enhance memory.

\section{Outcomes}

The primary interest of this review is the delivery and content characteristics of CBT for residential aged care settings. Delivery characteristics refer to the (1) frequency, (2) duration, and (3) mode (group vs individual) of treatment, as well as to (4) whether others were involved in the delivery of treatment (eg, nurses, other facility staff members, and family members), and (5) whether treatment was delivered alone or in combination with other interventions. In contrast, the content characteristics refer to the therapeutic techniques used (eg, behavioral activation and cognitive restructuring). In addition, information on stakeholders' reactions to these interventions will be described; such outcomes (whether assessed by a clinician, self-report, or an informant such as a staff member) will include participants' satisfaction with the CBT intervention, staff members' appraisal of the program, uptake rate, and attrition rate. Furthermore, these outcomes must be assessed with standardized, valid measures.

\section{Setting}

In this review, we will include studies conducted in residential care facilities and exclude those conducted only with community-dwelling older adults. Settings that are considered residential care facilities comprise nursing homes, aged care homes, residential aged care, and other communal living arrangements for older adults, where staff are employed to assist the residents with activities of daily living. Of note, studies conducted in retirement villages or retirement homes or hostels, where staff are only employed as on-site managers but do not provide care will be excluded.

\section{Types of Studies}

We will include empirical, quantitative studies that fulfill the criteria mentioned above. These studies could be randomized or quasi-randomized controlled trials, clinical controlled trials, cluster-randomized trials, cross-over trials, or case studies. However, commentaries and theoretical papers that describe protocols that have not been applied in residential aged care settings will be excluded.

\section{Report Characteristics}

We will include full-text papers written in English with no restrictions on the geographical location or year of publication.

\section{Search Methods for Identifying Studies}

We will be conducting a systematic search of the following databases to identify published studies: the Cochrane Library (including the Cochrane Central Register of Controlled Trials (CENTRAL) and the specialized registers of the Common Mental Disorders Group and the Dementia and Cognitive Improvement Group), Medical Literature Analysis and Retrieval System Online (MEDLINE), EMBASE, PubMed, PsycINFO, Cumulative Index of Nursing and Allied Health Literature 
the delivery and content of CBT within such settings to facilitate further development of such treatments across the sector.

\section{Acknowledgments}

This project was completed for partial fulfillment of the requirements for a Master of Psychology degree. No external funding was received.

\section{Authors' Contributions}

PC and SB devised the protocol. PC wrote the manuscript with inputs from other authors. All authors read and approved the final manuscript.

\section{Conflicts of Interest}

None declared.

\section{References}

1. Seitz D, Purandare N, Conn D. Prevalence of psychiatric disorders among older adults in long-term care homes: a systematic review. Int Psychogeriatr 2010 Nov;22(7):1025-1039. [doi: 10.1017/S1041610210000608] [Medline: 20522279]

2. Creighton AS, Davison TE, Kissane DW. The prevalence of anxiety among older adults in nursing homes and other residential aged care facilities: a systematic review. Int J Geriatr Psychiatry 2016 Jun;31(6):555-566. [doi: 10.1002/gps.4378] [Medline: 26552603]

3. Brownie S, Horstmanshof L, Garbutt R. Factors that impact residents' transition and psychological adjustment to long-term aged care: a systematic literature review. Int J Nurs Stud 2014 Dec;51(12):1654-1666. [doi: 10.1016/j.ijnurstu.2014.04.011] [Medline: 24813582]

4. Sury L, Burns K, Brodaty H. Moving in: adjustment of people living with dementia going into a nursing home and their families. Int Psychogeriatr 2013 Jun;25(6):867-876. [doi: 10.1017/S1041610213000057] [Medline: 23425369]

5. Davison TE, McCabe MP, Knight T, Mellor D. Biopsychosocial factors related to depression in aged care residents. J Affect Disord 2012 Dec 15;142(1-3):290-296. [doi: 10.1016/j.jad.2012.05.019] [Medline: 22901400]

6. Jongenelis K, Pot AM, Eisses AMH, Beekman ATF, Kluiter H, Ribbe MW. Prevalence and risk indicators of depression in elderly nursing home patients: the AGED study. J Affect Disord 2004 Dec;83(2-3):135-142. [doi: 10.1016/j.jad.2004.06.001] [Medline: 15555706 ]

7. Smalbrugge M, Pot AM, Jongenelis K, Beekman ATF, Eefsting JA. Prevalence and correlates of anxiety among nursing home patients. J Affect Disord 2005 Oct;88(2):145-153. [doi: 10.1016/j.jad.2005.06.006] [Medline: 16122811]

8. Baltes MM. The Many Faces of Dependency in Old Age. New York: Cambridge University Press; 1996.

9. Baltes MM, Reisenzein R. The social world in long-term care institutions: Psychosocial control toward dependency? In: Baltes MM, Baltes PB, editors. The Psychology of Control and Aging. Hillsdale, NJ: Lawrence Erlbaum Associates; 1986:315-343.

10. Wells Y, Bhar S, Kinsella G, Kowalski C, Merkes M, Patchett A, et al. What Works to Promote Emotional Wellbeing in Older People: A Guide for Aged Care Staff Working in Community or Residential Care Settings. Melbourne, Australia: Beyondblue; 2014.

11. Wilson K, Mottram PG, Vassilas C. Psychotherapeutic treatments for older depressed people. Cochrane Database Syst Rev 2008 Jan 23(1):CD004853. [doi: 10.1002/14651858.CD004853.pub2] [Medline: 18254062]

12. Hendriks GJ, Oude Voshaar RC, Keijsers GPJ, Hoogduin CAL, van Balkom AJ. Cognitive-behavioural therapy for late-life anxiety disorders: a systematic review and meta-analysis. Acta Psychiatr Scand 2008 Jun;117(6):403-411. [doi: 10.1111/j.1600-0447.2008.01190.x] [Medline: 18479316]

13. Gould RL, Coulson MC, Howard RJ. Efficacy of cognitive behavioral therapy for anxiety disorders in older people: a meta-analysis and meta-regression of randomized controlled trials. J Am Geriatr Soc 2012 Feb;60(2):218-292. [doi: 10.1111/j.1532-5415.2011.03824.x] [Medline: 22283717]

14. Cody RA, Drysdale K. The Effects of Psychotherapy on Reducing Depression in Residential Aged Care: A Meta-Analytic Review. Clin Gerontol 2013 Jan;36(1):46-69. [doi: 10.1080/07317115.2012.731474]

15. Anderson K, Wickramariyaratne T, Blair A. A feasibility study of group-based cognitive behaviour therapy for older adults in residential care. Clin Psychol 2016 Dec 15. [doi: 10.1111/cp.12109]

16. Blair AC, Bird MJ. A pilot trial of psychological therapy groups for the very old in residential care: Clinical and logistical issues. Clin Psychol 2013 Nov 05;20(2):68-79. [doi: 10.1111/cp.12031]

17. Curyto KJ, Trevino KM, Ogland-Hand S, Lichtenberg P. Evidence-based treatments for behavioral disturbances in long-term care. In: Scogin F, Shah A, editors. Making Evidence-Based Psychological Treatments Work with Older Adults. Washington, DC: American Psychological Association; 2012:167-223. 
18. Hyer L, Yeager CA, Hilton N, Sacks A. Group, individual, and staff therapy: an efficient and effective cognitive behavioral therapy in long-term care. Am J Alzheimers Dis Other Demen 2008;23(6):528-539. [doi: 10.1177/1533317508323571] [Medline: 19001352]

19. Meeks S, Van HK, Schoenbachler B, Looney SW. BE-ACTIV for depression in nursing homes: primary outcomes of a randomized clinical trial. J Gerontol B Psychol Sci Soc Sci 2015 Jan;70(1):13-23 [FREE Full text] [doi:

10.1093/geronb/gbu026] [Medline: 24691156]

20. Kishita N, Laidlaw K. Cognitive behaviour therapy for generalized anxiety disorder: Is CBT equally efficacious in adults of working age and older adults? Clin Psychol Rev 2017 Mar;52:124-136. [doi: 10.1016/j.cpr.2017.01.003] [Medline: 28119196]

21. Shamseer L, Moher D, Clarke M, Ghersi D, Liberati A, Petticrew M, PRISMA-P Group. Preferred reporting items for systematic review and meta-analysis protocols (PRISMA-P) 2015: elaboration and explanation. BMJ 2015 Dec 02;350:g7647 [FREE Full text] [Medline: 25555855]

22. Moher D, Liberati A, Tetzlaff J, Altman DG, PRISMA Group. Preferred reporting items for systematic reviews and meta-analyses: the PRISMA statement. BMJ 2009 Jul 21;339:b2535 [FREE Full text] [Medline: 19622551]

23. Higgins JPT, Green S, editors. Cochrane Handbook for Systematic Reviews of Interventions Version 5.1.0. Melbourne, Australia: The Cochrane Collaboration; 2011.

24. Downs SH, Black N. The feasibility of creating a checklist for the assessment of the methodological quality both of randomised and non-randomised studies of health care interventions. J Epidemiol Community Health 1998 Jun;52(6):377-384 [FREE Full text] [Medline: 9764259]
Abbreviations
CBT: cognitive behavioral therapy
CENTRAL: Cochrane Central Register of Controlled Trials
CINAHL: Cumulative Index of Nursing and Allied Health Literature
EBSCO: Elton B Stephens CO (company)
ICTRP: International Clinical Trials Registry Platform
MMSE: Mini Mental State Examination
PRISMA: Preferred Reporting Items for Systematic Review and Meta-Analysis

\author{
Edited by G Eysenbach; submitted 21.01.18; peer-reviewed by H Jayaram, $R$ Slikboer, J Lambert; comments to author 29.03.18; \\ revised version received 30.04.18; accepted 08.05.18; published 04.07.18 \\ Please cite as: \\ Chan P, Bhar S, Davison TE, Doyle C, Knight BG, Koder D, Laidlaw K, Pachana NA, Wells Y, Wuthrich VM \\ Characteristics of Cognitive Behavioral Therapy for Older Adults Living in Residential Care: Protocol for a Systematic Review \\ JMIR Res Protoc 2018;7(7):e164 \\ URL: http://www.researchprotocols.org/2018/7/e164/ \\ doi: $10.2196 /$ resprot. 9902 \\ PMID: 29973335
}

CPhoebe Chan, Sunil Bhar, Tanya E. Davison, Colleen Doyle, Bob G. Knight, Deborah Koder, Kenneth Laidlaw, Nancy A. Pachana, Yvonne Wells, Viviana M. Wuthrich. Originally published in JMIR Research Protocols (http://www.researchprotocols.org), 04.07.2018. This is an open-access article distributed under the terms of the Creative Commons Attribution License (https://creativecommons.org/licenses/by/4.0/), which permits unrestricted use, distribution, and reproduction in any medium, provided the original work, first published in JMIR Research Protocols, is properly cited. The complete bibliographic information, a link to the original publication on http://www.researchprotocols.org, as well as this copyright and license information must be included. 